\title{
TISSUE DOPPLER ONE-SEGMENT STRAIN AND STRAIN-RATE ANALYSIS ARE MORE SENSITIVE THAN FRACTIONAL SHORTENING FOR ASSESSING REDUCED MYOCARDIAL FUNCTION AFTER BIRTH ASPHYXIA
}

\author{
E. Nestaas ${ }^{1}$, A. Støylen ${ }^{2,3}$, D. Fugelseth ${ }^{4,5}$ \\ ${ }^{1}$ Department of Paediatrics, Vestfold Hospital Trust, Tønsberg, ${ }^{2}$ Department of Cardiology, St. Olavs \\ Hospital, ${ }^{3}$ Department of Circulation and Medical Imaging (ISB), Faculty of Medicine, Norwegian \\ University of Science and Technology, Trondheim, ${ }^{4}$ Department of Paediatrics, Oslo University Hospital, \\ Ulleval, ${ }^{5}$ Institute of Clinical Medicine, Faculty of Medicine, University of Oslo, Oslo, Norway
}

Background and aims: Strain and strain-rate are heart function indices not influenzed by heart size. We compared systolic and diastolic heart function assessed by one-segment strain and strain-rate analysis against heart function assessed by the conventional index Fractional Shortening (\%FS), between healthy and asphyxiated term neonates.

Methods: Healthy and asphyxiated term neonates were examined daily on day 1-3 of life. At each examination we measured \% $\mathrm{FS}$ and in nine heart segments the deformation indices peak systolic strain (PSS), peak systolic strain-rate (PSSR), early diastolic strain-rate (ESR) and strain-rate during atrial systole (ASR).

Results: Analyses were performed in 138 examinations from 48 healthy term neonates (gestational age (GA) $41(37,41)$ weeks (median, range), 5 min Apgar score $9(8,10))$ and 53 examinations from 20 asphyxiated neonates (GA $40.5(37,42)$ weeks, 5 min Apgar score $5(4,6))$. PSS, PSSR, ESR and ASR were all reduced (closer to zero) in asphyxiated neonates than in healthy term neonates (PSS $-17.8 \%(-19.0,-16.7)$ (mean and 95\% confidence interval) vs. $-21.2 \%(-21.7,-20.7)$, PSSR $-1.44 / \mathrm{s}(-1.52,-1.34)$ vs. $-1.61 / \mathrm{s}(-1.66,-1.56)$, ESR 1.72/s $(1.61,1.84)$ vs. $2.03 / \mathrm{s}(1.91,2.14)$ and ASR 1.91/s $(1.73,2.10)$ vs. $2.27 / \mathrm{s}(2.17,2.38), \mathrm{p}<0.003)$. $\%$ FS was similar in the asphyxiated $(29.1 \%(26.9,31.2))$ and healthy term neonates $(29.0 \%(27.9,30.0))$ $(\mathrm{p}=0.922)$.

Conclusions: Both the systolic and diastolic cardiac function indices were reduced in the asphyxiated neonates. These cardiac function indices were more sensitive than Fractional Shortening for assessing the impaired myocardial function in asphyxiated term neonates. 